\title{
THE TREATMENT OF SYPHILIS WITH PENICILLIN*
}

BY

JOSEPH EARLE MOORE

I shall try to approach the subject of the treatment of syphilis with penicillin not so much from a statistical point of view, but rather from the point of view of some fundamental questions of the biology of syphilitic infection and its treatment. I propose to give a brief account of the American experience with penicillin.

Treatment has been conducted on an organized co-operative basis, first under the auspices of the Committee on Medical Research, guided by the Sub-Committee on Venereal Diseases of the National Research Council, and, since January 1, 1946, under the Research Grants Division, National Institute of Health, of the United States Public Health Service. Co-operating in the investigation originally were the U.S. Army, Navy, and Public Health Services, and some twenty-five civilian clinics. The information gained from each of these clinics was reported on standard forms to a central statistical unit where they were analysed by the Department of Bio-statistics of the Johns Hopkins School of Hygiene and Public Health. We now have data on some 40,000 patients with early syphilis, and a smaller but substantial number of persons with various phases of late syphilitic infection. Some questions seem to have been answered; but even more fundamental questions have appeared as a result of the investigation. To use a military phrase, some tactical advances have been made, perhaps, but we are faced in the long run with a number of important strategic questions to which we do not, as yet, know the final answers.

\section{Chemistry of Penicillin in the Treatment of Syphilis}

I would 'begin with a brief discussion of the chemistry of penicillin as applied to the treatment

\footnotetext{
- This address, and those at pp. 9 and 11 of this issue, and the discussion following them, formed the substance of a joint meeting of discussion following them, formed the substance of a joint meeting of
the Section of Experimental Medicine and Therapeutics, Royal Society
of Medicine, and the Medical Society for the Study of Venereal of Medicine, and the Medical Society for the Study of Venereal
Disease, on July 17, 1947. The material is published by permission
of the Honorary Editors of the Proceedings of the Royal Society of Medicin. A short account appears in the Proceedings, vol.40, p.811.
}

of syphilis. In addition to the co-operating clinics involved in the American experiment, there are also eight laboratories which have worked on a co-operative basis on the study of penicillin in syphilis in rabbits. From their work, it appears that of the identified penicillin species, penicillin $G$ is superior in treponemicidal effect to $X$ or $K$. This is in contrast to the bactericidal effect of these species against certain other organisms. Penicillin $\mathbf{X}$ is from two to ten times superior to $\mathrm{G}$ in gonococcal, staphylococcal, and pneumococcal infections. In syphilitic rabbits, however, $G$ is about six times as effective as $F$ or $X$, and about twenty times superior to penicillin K. From the practical standpoint, this is fortunate, since commercial penicillin, almost since its inception, has been composed predominantly of penicillin $G$; and $G$ is the easiest species to prepare in relatively pure crystalline form. The other penicillin species, $F$, dihydro $F, X$, and $K$, have been available in pure form only in very small amounts for experimental study.

It is known that commercial penicillin also contains impurities, some of which either possess in themselves or enhance both treponemicidal and bactericidal action. These impurities have not yet been definitely identified nor their relative merits contrasted with the known penicillin species.

While new and modified penicillin species have not yet been developed in large enough amounts to permit even their experimental study, other antibiotic agents, in particular streptomycin and bacitracin, have not been shown to equal the effect of penicillin $\mathbf{G}$.

Penicillin cures syphilis in rabbits whether the animals are treated early (within six weeks after infection) or late (six months or later after infection). The time-dose relationship may be so adjusted that 100 per cent. of rabbits are cured, judging cure by the criterion of lymph-node transfer six months after treatment. Many small doses are more effective than one or a few large doses. The curative dose is related also to the duration of infection. In animals treated within four hours to four days after 


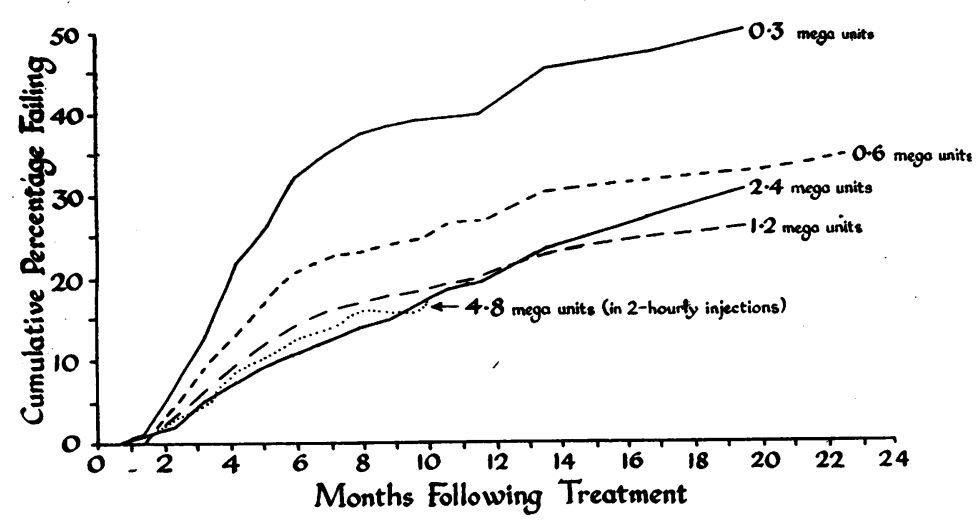

Fig. 1.-Cumulative failure rates by total dose of penicillin. Penicillin only : every 3 hours for seven or eight days.

\section{Analysis of Some 40,000} Case Records

Passing to early acquired infection in man, if freedom from relapse and prolonged seronegativity up to three years after treatment indicate cure of early syphilis in man, then penicillin is also curative, but by no means so uniformly so as in the experimental animal. The co-operative study of penicillin, which is based on some 40,000 records, has attempted to analyse a considerable number of variables. The major ones are total dosage of peni- inoculation, therefore, before the appearance of a chancre, far less penicillin is required (total dosage) than if treatment is delayed for six weeks or longer. The results in animals are likewise related to the size of the inoculum : animals treated soon after the injection of from two to twenty organisms require a smaller total dose for cure than if the inoculum is a large one. Cure is accomplished within the actual period of treatment, since treated animals with healing chancres still present may be reinfected as soon as four days after the completion of treatment.

The relationship of the curative dose of penicillin to the duration of the infection and to the size of the inoculum, that is, the facts that a relatively very small total dose of penicillin is necessary if the animals are treated within four hours to four days after inoculation, and if they are inoculated with small doses, has raised the possibility of the prophylactic use of penicillin by means of oral administration. Both the U.S. Army and U.S. Navy now have in progress controlled experiments in selected units in which 500,000 units of penicillin administered by mouth in personnel sexually exposed is being tried for prophylactic effect.

It is important to note that in experimental animals, neither in vitro against cultured nonpathogenic strains of spirochætes, nor in vivo, has there appeared any definite evidence of penicillin resistance.

In animals the effect of penicillin is apparently enhanced by the simultaneous administration of oxophenarsine hydrochloride (mapharside). The effect of penicillin is likewise enhanced in an additive sense by the simultaneous administration of bismuth. The drug is more effective when administered at fever temperatures than at normal body temperatures. cillin administered, the duration of treatment, the interval between injections of penicillin, the effect of addition of other chemotherapeutic agents such as arsenic, bismuth, or fever to penicillin, the relationship of the results to the potency of the penicillin product employed, the relationship of the results to the duration of infection. Patients have been classified as failures if at any time after treatment there was evidence of clinical or serologic relapse or reinfection. It is felt in the U.S.A. that it is clinically impossible to differentiate reinfection from relapse, and in view of this, even though the presumption of reinfection was very strong, all such patients were classified as treatment failures. To the extent that there is a predominance of actual reinfection over relapse, the results are better than indicated. Serologic relapse includes not only patients who relapse after having become seronegative, but also those in whom serologic titre drops to a low level followed by a subsequent rise in titre. Patients have also been classified as failures if at

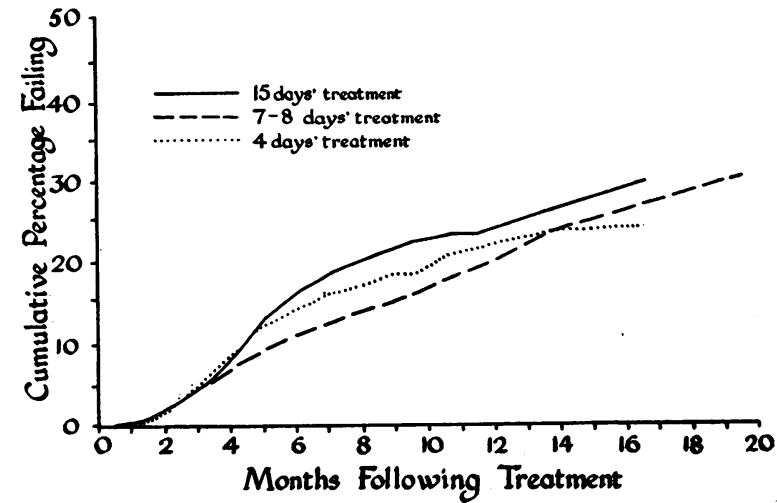

FIG. 2.-Cumulative failure rates by duration. Penicillin only: every three hours, $2 \cdot 4$ million units. 


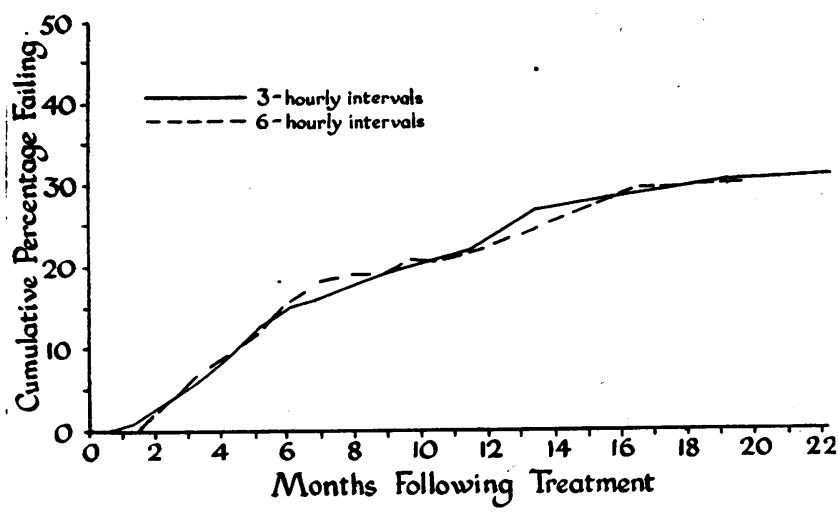

Fig. 3.-Cumulative failure rates by interval. Penicillin only : for four days, $1 \cdot 2$ million units.

the end of one year after treatment reagin persists in the blood, that is, if they are sero-resistant.

Cumulative Failure Rate.-Fig. 1 shows the cumulative failure rate by total doses of penicillin. This is in patients with all types of early syphilis. The penicillin was administered every three hours for seven to eight days. There is an obvious relationship between total dose and failure rate; when the total dose was 300,000 units the failure rate at the end of twenty-two months approximates 50 per cent. ; with 600,000 units it drops to 35 per cent., but when the dose reaches 1.2 to 4.8 mega units there is no appreciable difference between the failure rate at the end of one year.*

Comparison by Range of Treatment.-Fig. 2 shows a comparison with a total range of treatment of four days, seven and a half days, and fifteen days. In this case the treatment system -employed was penicillin alone administered intramuscularly every three hours to a total of 2.4 million units. Involved in this analysis are some forty-five different treatment schedules, and I have selected only illustrative examples.

Comparison by Interval between Doses.-Fig. 3 shows the results by intervals between individual doses; the intervals being every three hours and every six hours, the total duration of treatment remaining standard at four days, and the total dose remaining standard at 1.2 million units. Using penicillin in aqueous solution there is no difference

- The clinical experiment with penicillin was designed to be on a quantitative basis. In that respect, however, results must be taken with a grain of salt. The manufacturers are required to ampoule penicillin in such a manner that the unit content stated on the ampoule may not be decreased by more than 15 per cent. An ampoule said to may not be decreased by more than 15 per cent. An ampoule said to
contain 100,000 units must contain a minimum of 85,000 units or be discarded. In order to avoid this, manufacturers commonly place an excess of penicillin in the ampoule. When we speak of quantitative results utilizing commercial penicillin, as contrasted with crystalline penicillin $G$, there is a quantitative error of between -15 per cent. and +40 per cent. in the result whether the interval between injections is three hours or six hours. We have not employed aqueous penicillin at less frequent intervals than six hours except in a small group of patients who were given 25 million units by continuous intravenous drip in one day. In them the failure rate approximated about 60 per cent. at the end of six months.

Impurity versus Purity.-Fig. 4 is calculated to examine the question whether impure crude penicillin is more effective than a highly purified product. We have information as to potency in terms of units per milligramme of all the penicillin involved in this study, beginning with the impure penicillin of 1943 (about 300 $\mathrm{u} / \mathrm{mg}$.) up to our present level of unitage (about $1,500 \mathrm{u} / \mathrm{mg}$.). There appears to be no statistically significant difference as between 'penicillin of relatively low potency and penicillin of comparatively higher potency, 900 units or more per milligramme.

Penicillin with Adjuvants.-Fig. 5 attempts to compare the results in patients given an identical amount of penicillin for an identical period, that is, 1.2 million units of penicillin for seven and a half days, combined with three different adjuvants; patients treated with penicillin plus arsenic, those given penicillin plus bismuth, and two groups of patients treated with penicillin plus fever (one group with four days' fever, the other with seven). There appears to be no material advantage in human beings to a combination of penicillin with bismuth or arsenic, in the dosages and within the time limits in which these drugs were used. A total dose of $480 \mathrm{mg}$. of auxiliary mapharsene chloride was given; of bismuth, $1,000 \mathrm{mg}$. These are doses

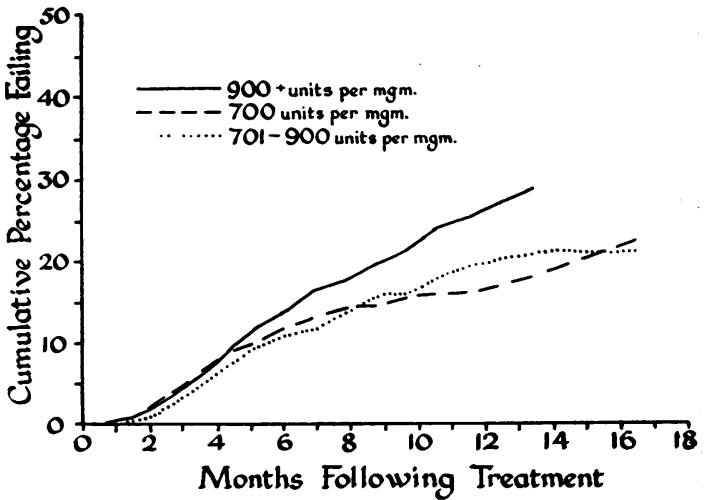

Fig. 4.-Cumulative failure rates by potency of penicillin. Penicillin only, $2 \cdot 4$ million units. 


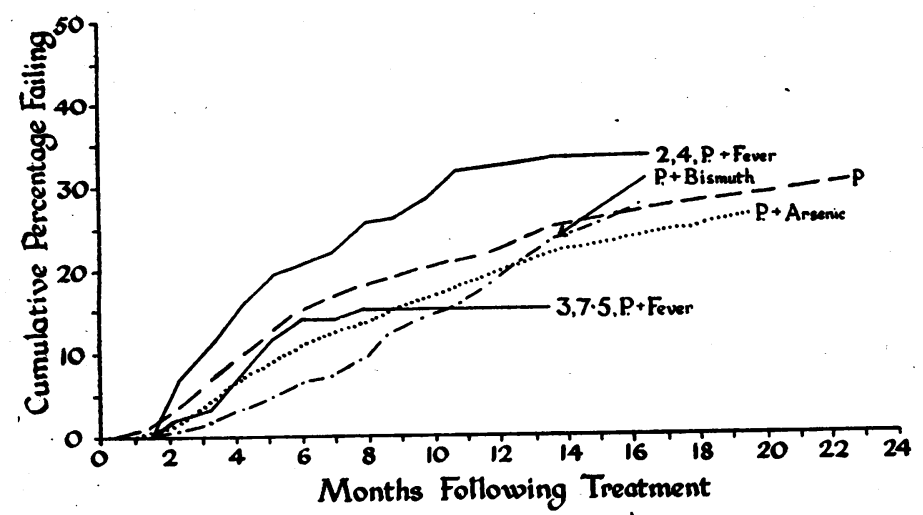

Fig. 5.-Cumulative failure rates by treatment agent ; 1.2 million units of penicillin.

known to be in themselves non-curative and subject to a high relapse rate.

Duration of Infection.-Fig. 6 illustrates the difference in results based on the duration of infection, expressed in terms of days dating from the first appearance of the original lesion. These we have deliberately selected as being a rather more accurate differentiation of early syphilis than the clinical classification of sero-negative and seropositive primary and early secondary syphilis. Females and coloured males were excluded from this tabulation because of the uncertainty in them of the first appearance of the chancre. To the time periods indicated must be added the approximate three weeks' incubation period which precedes the appearance of the lesion. There is an obvious advantage in giving treatment early; in patients treated within the first twenty-seven days the results are materially better than when treatment is delayed until lesions have been present for from four to seven weeks, and still better than when lesions have been present for roughly eight weeks or longer. The data are roughly separable into the clinical groups of sero-negative primary syphilis (less than seven days), and early secondary syphilis (more than fifty-six days).

Ambulatory Treatment.-We have been most interested in the United States in the possibility of developing an ambulatory method of treatment. This seems impossible to accomplish with penicillin in aqueous solution without the use of prohibitively large doses and at excessive cost. We have therefore concentrated on a study of absorption-retarding methods, the most advantageous of which is the use of penicillin in peanut oilbeeswax, a formula involving the suspension of penicillin in peanut oil which contains 4.8 per cent. of purified beeswax. Penicillin in beeswax may be administered once a day rather than every few hours since one gets a detectable blood revel from a single dose $(600,000$ units) which lasts twenty to twenty-four hours. Patients have been so treated with three different systems, one with the administration of 600,000 units of penicillin daily for eight days, another with daily injections of 600,000 units for sixteen days, and the third by twice-weekly injections given over eight weeks to a total of sixteen injections.

The results of the penicillin-peanut oil-beeswax systems of treatment are relatively indistinguishable. Fig. 7 compares the results, in primary syphilis, of 1.2 million units aqueous penicillin given by four different treatment systems, 2.4 million units given in aqueous solution by two different schedules, and 4.8 million units given in peanut oil-beeswax. The data suggest that at the end of twelve to twenty-four months the results from penicillin in peanut oilbeeswax are as good as, and may be better than, would be expected from penicillin in aqueous solution. This is being confirmed by still further observation.

Types of Failure Compared.-Fig. 8 shows the types of failure observed with varying treatment systems. We were interested in attempting to determine whether the incidence of relapse or reinfection was higher after one treatment system than after another. The chart compares four different systems. The incidence of clinical relapse,

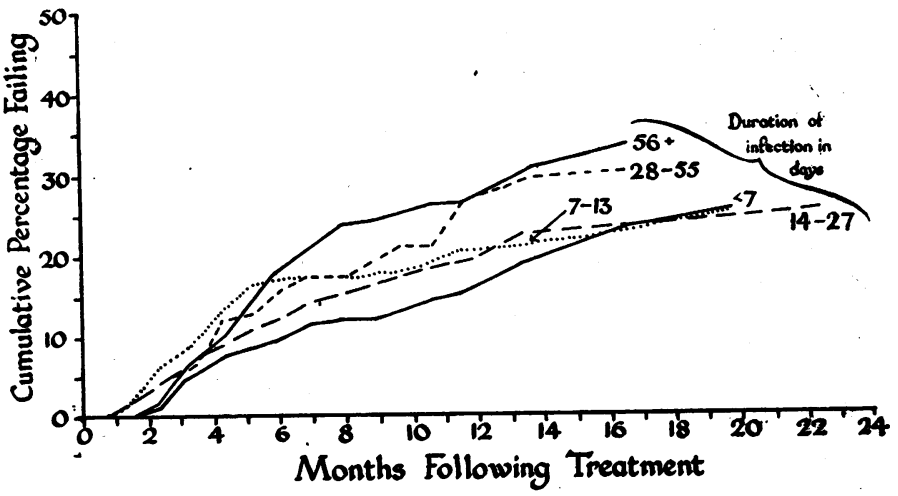

FIG. 6.-Cumulative failure rates by duration of disease. Penicillin only, $1 \cdot 2$ million units. 


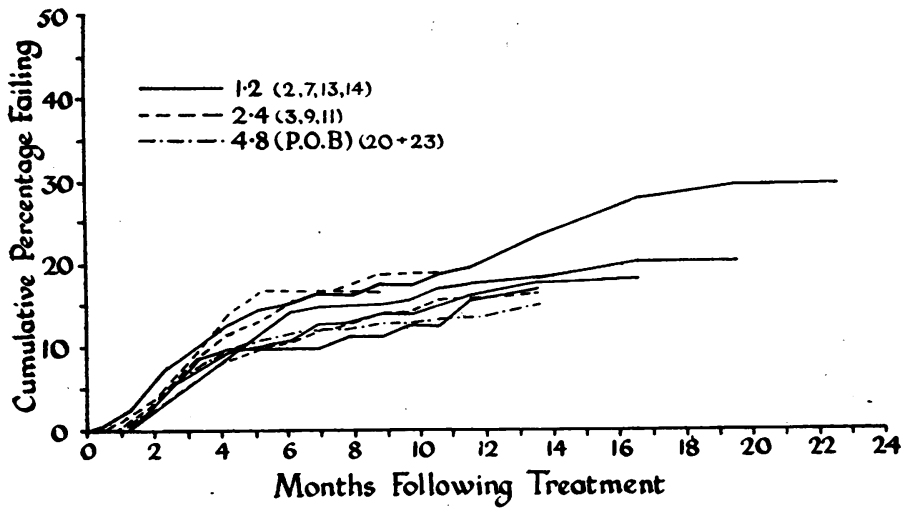

Fig. 7.-Cumulative failure rates by total dose of penicillin and vehicle penicillin only; primary syphilis.

syphilis in that an adjuvant total dose of bismuth adequate to prevent relapses over the first nine to twelve months may allow the patient to develop enough immunity so that he is infectious for others for a shorter time than would otherwise be the case.

\section{Results of Analysis}

These results on the whole indicate that the failure rate of treatment with penicillin in patients with early syphilis after eighteen to twenty-four months of observation is, with the treatment systems which we have employed, in the general range of 25 to 35 per cent. This failure rate is not materially improved including reinfection, is about the same regardless of how penicillin was employed, whether the dosage was low or relatively high, and whether the drug was or was not combined with metal chemotherapy.

The time of appearance of relapse when penicillin is combined with bismuth is slightly later than when the drug is used alone or with arsenic. This may be of some importance to the mass treatment of upon by the addition of arsenic or of bismuth or of arsenic plus bismuth in the dosages and time periods in which they have been used. The results are likewise not materially improved upon by fever therapy. These are results which are substantially worse than the best obtainable by metal chemotherapy without penicillin, where the failure rate may be as low as 3 to 10 per cent., provided the

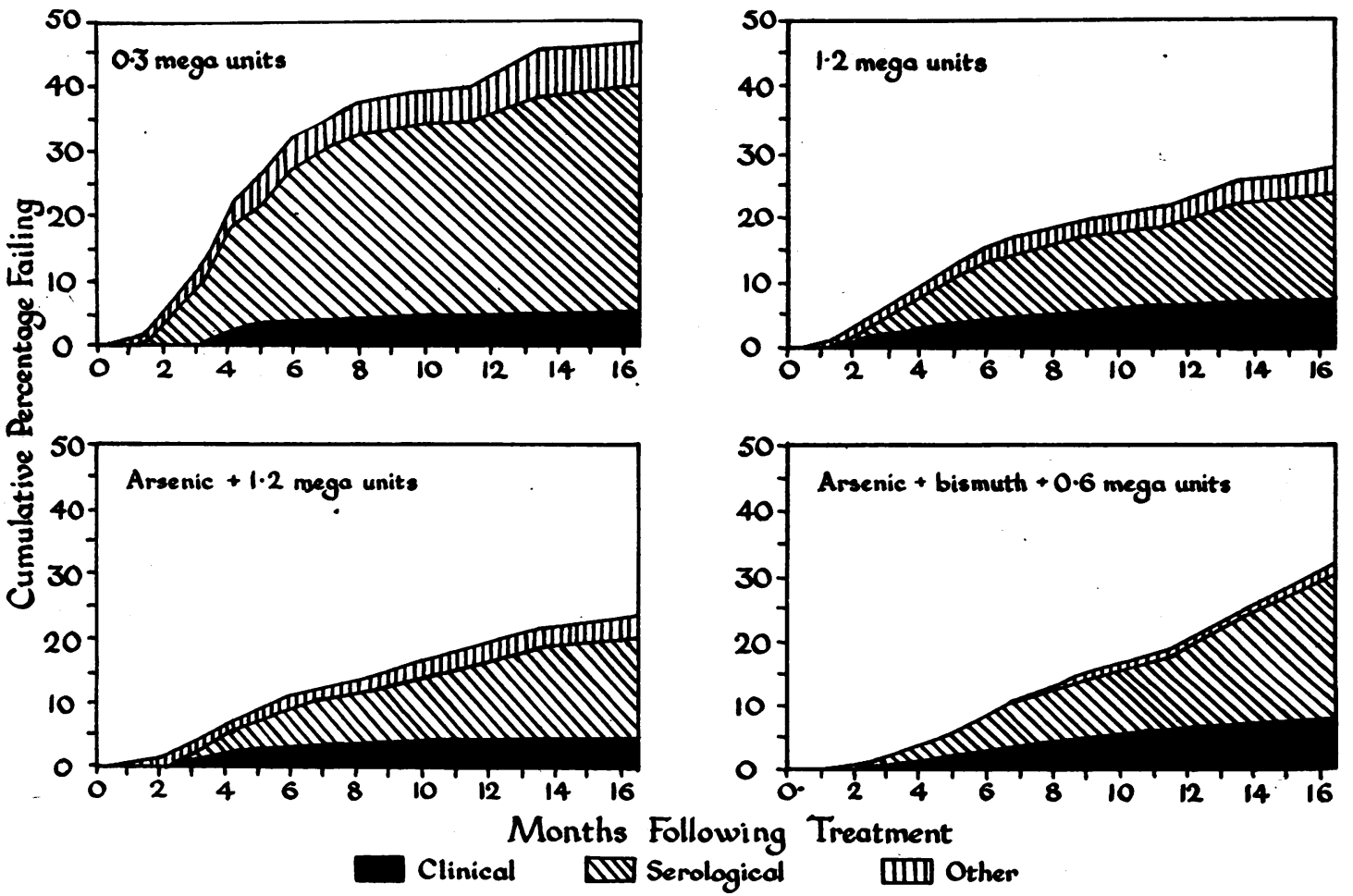

C

FIG. 8.-Cumulative failure rates by type of failure. 
patient completes treatment and is not made seriously ill or killed as a result of it. The disadvantages of metal chemotherapy lie in the facts that, if treatment is compressed within a brief time period, the mortality rate from treatment per se is excessive, whereas if it is prolonged for the sake of safety, comparatively few patients can be held to the conclusion of treatment. Penicillin offers the advantage of complete safety when used within the - limits of practicality, which is, with penicillin in aqueous solution about fifteen days. This is the maximum time for which we can hold patients in hospital. On the other hand, the results are such as to indicate that for the individual patient they may be poor, that is, less than can be accomplished with metal chemotherapy alone.

The data given apply only to commercial penicillin. Results with crystalline penicillin $G$, now under study, will not be available for another twelve to eighteen months. It takes a maximum of eighteen months of observation of a series of about 1,000 treated cases to arrive at a reasonable conclusion with regard to the results. Information with regard to the absorption-delaying method of penicillin in peanut oil-beeswax is preliminary in the sense that it does not extend beyond twelve months. However, within the total dosage range of 4.8 and 9.6 million units, and within the time limits of twice daily injections, or injections once or twice a week for eight weeks, the results appear to be as good as with the drug in aqueous solution.

Penicillin resistance has not been observed in syphilis in man.

Some Fundamental Questions.-This information raises a number of fundamental questions, most of which are related to the biology of syphilitic infection. Why is early syphilis uniformly curable in the rabbit, whereas in man, no matter what the system of treatment employed be, there is a substantial residue of failure? Is this related to the number of treponemes to be destroyed-few in the rabbit but many in man? Is it related to the factor of tissue localization in man but not in animals ? Organisms may lodge in tissues to which the therapeutic agents do not penetrate. We know that treponemes do lodge in the nervous system, to which penicillin does not penetrate as well as elsewhere. But there is a curious difficulty here which I shall point out in dealing with the uses of penicillin in neurosyphilis. Is this difference in animals and man due to factors of immunity? Is man dependent on the development of resistance against the few treponemes not destroyed in the first massive attack ? Why do not the phenomena observed in the laboratory, as to the added effect of arsenic, bismuth, and fever, likewise hold good in man? What is the significance of low titre sero-resistance after penicillin, that is, the persistence of a small quantity of reagin in the blood for a long period of time? What is the source of reagin in the blood? Is it related to the total mass of syphilitic lesions? Does it represent persistence of treponemes or the tissue reaction which they provoke ; or does it represent immunity persisting after cure? Can a man be successfully re-inoculated while still sero-positive ? Can any criteria be evolved which will serve to differentiate relapse and reinfection?

A great deal of information has accumulated which indicates that with other bacteria penicillin is effective only when the organisms are in a rapidly dividing state. Is it possible that the worse results in late secondary syphilis depend on this factor? Just what is the relationship of the time-dose factor to the rate of multiplication of the treponeme? Since pathogenic $T$. pallidum has never been successfully grown, it is not possible to solve these problems in vitro. In contrast to most pathogenic bacteria, which divide within vitro or vivo within a period of hours or days, the time necessary for multiplication of spirochætes to the point necessary to provoke tissue reaction may be measured in weeks, months, or even years. This multiplication rate must be important. If all the treponemes are not killed by the first massive therapeutic dose, is it possible that repeated courses of penicillin for a year or so, or twice weekly injections of penicillin in peanut oil-beeswax for six to twelve months might be more effective than a single short course?

In view of the fact that the incidence of fresh infections has not decreased, at least in the United States, but has apparently actually increased, is it possible that this is due, not as we have tended to think, to the influence of war, but instead to the possibility that intensive treatment methods, including penicillin, applied to millions of persons have tended to increase the period of infectivity of the infected population rather than to decrease it ?

Does prolonged sero-negativity in man indicate cure ? If so, how long must sero-negativity continue before the patient can be discharged from further investigation?

These are only a few of the fundamental questions to which we do not yet know the answer.

Prenatal Syphilis.-Now to pass to an even more curious anomaly in the use of penicillin in the treatment of syphilis. In contrast to a failure rate of 25 per cent. or more in patients with acquired early syphilis, penicillin is spectacularly and nearly completely successful in the prevention of prenatal syphilis by the treatment of the pregnant syphilitic 
mother. The failure rate in the infant is only 1 or 2 per cent. The total dose and duration of treatment are the same as in early syphilis. This paradox raises another fundamental biological issue. Why should foetal infection, which is often massive and overwhelming as contrasted with the acquired disease in adults, be so readily and completely curable as the result of a tissue concentration of penicillin substantially lower' than that in the maternal body? Why is it that a pregnant woman with secondary syphilis is not cured herself by penicillin, although her fotus is favourably affected even if treatment is not begun until the eighth or ninth month?

Infantile Congenital Syphilis.-Almost the same problem is raised by the use of penicillin in infantile congenital syphilis. Here penicillin has not reduced the mortality rate, which remains at 10 or 15 per cent. Most of the deaths in penicillin-treated infants are not due to syphilis per se nor to the Herxheimer reaction, but to other infections or nutritional disturbances. Penicillin may actually make worse the infantile diarrhœa due to Salmonella infections. However in syphilitic infants who survive after treatment the failure rate is substantially lower than the adult rate, and clinical relapse is only about one-sixth as frequent. Why should this be? If, as has been suggested with acquired early syphilis, the failure rate may be influenced by the number of treponemes in the body, why do infants, whose tissues may be swarming with organisms, do better than adults in whom spirochates are relatively few? Is this due to differences in infantile and adult tissue localization, or is it due to unidentified factors of immunity which may vary in the infant as contrasted with the adult?

Efiect on Lesions and on Blood Tests.-In latent and late syphilis other than neurosyphilis, penicillin heals lesions if any are present but has no greater effect on the blood serological test than does any other form of treatment: Nothing can be said of eventual clinical outcome in these cases, since years of post-treatment observation are necessary to determine the point. In patients with latent syphilis, what matters is what happens to the patient, not what happens to his blood test; and it is necessary to follow large series of patients quite literally for a lifetime.

Cardiovascular Syphilis. - In cardiovascular syphilis the most that can be said about penicillin to date is that this form of treatment, whether initiated with large or small doses, appears to do no harm. The Herxheimer reaction, so-called therapeutic shock, need not be greatly feared. At the Johns
Hopkins Hospital we have now treated some fifty patients with saccular aneurysms or aortic regurgitation, dividing them into two groups, one of which was treated initially with 1,000 units gradually increased to a total therapeutic dosage, and the other was treated from the start with 50,000 to 100,000 units. In neither group was there evidence of damage to the patient which could be measured by clinical symptoms, electrocardiograms, sedimentation rates, temperature, leucocyte count, and so on. No one is yet prepared to offer any statement as to whether penicillin does people with cardiovascular syphilis any good, but since it heals visible lesions it should heal lesions which cannot be seen.

Late Syphilis.-There is here another curious and important difference between experimental animals and man. Rabbits with late syphilis appear to be as readily curable with penicillin as do those with early infections. A smaller total dose of penicillin, even, may be required to cure the animals with late than with early syphilis. Is late syphilis in man curable with this drug ? Only much further study will give us the information.

Syphilis of the Nervous System.-In syphilis of the nervous system, and regardless of the clinical syndrome present, there are profound and uniform effects from penicillin administered alone by the intramuscular route. Whether asymptomatic neurosyphilis, acute syphilitic meningitis, tabes dorsalis, or general paresis, cell count and protein content are reduced to normal in practically all cases; and this fact is apparent within a few weeks and in many cases within a few days. Colloidal tests tend towards normal as protein decreases. The Wassermann test of the spinal fluid is also favourably though more slowly influenced. The rate of reversal of this test depends on two identified factors: first, the duration of infection with syphilis ; and secondly, the original complement fixation titre of the fluid. The more strongly positive the fluid, the longer it takes for the Wassermann test to become negative. These evidences of laboratory improvement, regardless of clinical type of disease, persist at least for three years in about 90 per cent. of cases treated, only about 10 per cent. of the total showing a later return of fluid abnormalities. There is no need for the technically difficult administration of penicillin by the subdural or intrathecal route.

Clinically, penicillin appears to be superior to any form of metal chemotherapy. Regardless of the type of neurosyphilis, clinical improvement may be expected from penicillin to the extent to which symptoms and physical signs depend on inflammation rather than on degeneration. Neither penicillin 
nor any other form of treatment will restore dead brain cells or fibre tracts, although further destruction and clinical progression may be prevented.

In certain of the relatively benign forms of neurosyphilis, penicillin alone gives satisfactory results. In the more serious forms the combination of penicillin and fever from induced malaria may be superior, both from clinical and laboratory standards, to penicillin alone.

The time-dosage relationship in neurosyphilis is not so clearly established as in early syphilis. Most American observers believe that a large total dose is superior to smaller dosage and that ten to twenty days' treatment is better than a shorter duration.

The advent of penicillin has made it possible to accomplish as much or more in most cases of neurosyphilis as previously was obtainable with fever and several years of subsequent metal chemotherapy. Its immediate effects appear to be more striking in neurosyphilis than in early syphilis. Here is the anomaly to which I drew attention earlier. Available data indicate that penicillin penetrates the tissues of the nervous system in a concentration far lower than that of other tissues. If improvement depends on tissue concentration of the drug, why are results apparently better in neurosyphilis than in early syphilis in which the nervous system is spared? Are the good results permanent?

\section{Summing-Up}

In short, penicillin represents a major advance in syphilotherapy. There are curious and unexplained differences in its effect in various stages of syphilitic infection as it is now available. Whether the variable mixtures of commercial penicillin or crystalline penicillin $G$ is used, it probably represents only the first faltering steps in the antibiotic therapy of this disease. There is reason to expect that further studies may replace the present product with a new and more powerful antibiotic. Pending such a development, the organized study of penicillin has provided information within four years which otherwise would have required a generation to accumulate. More important, this co-operative study has re-emphasized that gaps in the knowledge of the fundamental biology of syphilitic infection exist which must be filled in before the perfect form of treatment of syphilis can be found. 